\title{
Hydroxylapatit als biomimetischer Wirkstoff für die Remineralisation von Zahnschmelz und Dentin
}

Joachim Enax, Helge-Otto Fabritius, Bennett T. Amaechi, Frederic Meyer

In der Mundhöhle herrscht ein dynamisches Gleichgewicht zwischen Demineralisation und Remineralisation des Zahnminerals. Dieses Gleichgewicht kann durch bakterielle Biofilme (Karies) oder säurehaltige Nahrungsmittel (Erosion) in Richtung der unerwünschten Demineralisation verschoben werden. Eine Möglichkeit, den Zahnschmelz und das Dentin mit einem Wirkstoff zu remineralisieren, ist die Verwendung von partikulärem Hydroxylapatit $\left[\mathrm{Ca}_{5}\left(\mathrm{PO}_{4}\right)_{3}(\mathrm{OH})\right]$.

Hydroxylapatit (HAP) ist ein gut verträgliches, biomimetisches Kalziumphosphatmineral und stellt damit im sauren Milieu eine potenzielle Quelle für Kalzium- und Phosphationen dar. Studien zeigen, dass sich HAP-Partikel an die Zahnoberfläche anlagern. Die Verwendung von HAP führt zu einer Remineralisation des demineralisierten Zahnschmelzes bis in tiefere Schichten, was unter Mundhöhlenbedingungen (In situ) gezeigt wurde. Im folgenden Artikel werden wissenschaftliche Belege in Form publizierter In-vivo-, In-situ- und In-vitro-Studien zusammengefasst.

\section{Demineralisation und Remineralisation des Zahnminerals/Kalziumphosphate in der Zahnpflege}

Der Zahnschmelz besitzt eine komplexe, hierarchisch organisierte Mikrostruktur aus Hydroxylapatitkristalliten $\left[\mathrm{Ca}_{5}\left(\mathrm{PO}_{4}\right)_{3}(\mathrm{OH})\right]$ und ist das härteste Gewebe im menschlichen Körper [1-4]. Neben anderen Ursachen (wie z.B. mechanischem Abrieb) kann er insbesondere durch Säuren geschädigt werden. Dies wird als Demineralisation bezeichnet [5]. Es ist bekannt, dass der kritische $\mathrm{pH}$-Wert von Schmelz 5,5 beträgt [6]. Allerdings ist nicht nur der $\mathrm{pH}$-Wert entscheidend für das Ausmaß der Demineralisation, sondern auch die Anwesenheit von Kalzium- und Phosphationen, die dieser durch Remineralisation entgegenwirken [6,7]. Aus diesem Grund stellt die Verwendung von Kalziumphosphaten (z. B. Hydroxylapatit $\left[\mathrm{Ca}_{5}\left(\mathrm{PO}_{4}\right)_{3}(\mathrm{OH})\right]$, $\beta$-Trikalziumphosphat $\left[\beta-\mathrm{Ca}_{3}\left(\mathrm{PO}_{4}\right)_{2}\right]$ und amorphen Kalziumphosphaten $\left.\left[\mathrm{Ca}_{\mathrm{x}}\left(\mathrm{PO}_{4}\right)_{\mathrm{y}} \cdot \mathrm{n} \mathrm{H}_{2} \mathrm{O}\right][3,8]\right)$ in Zahnpflegepräparaten einen vielversprechenden Ansatz zur Remineralisation dar.
Aus der Reihe der Kalziumphosphate besitzt Hydroxylapatit (HAP) die höchste Übereinstimmung mit den Schmelzkristalliten humaner Zähne und gilt deshalb als biomimetischer Wirkstoff [9-12]. Bei dem in Zahnpflegeprodukten verwendeten HAP, wie in einer detaillierten rasterelektronenmikroskopischen (REM) Untersuchung gezeigt, handelt es sich um stabile Mikrocluster (Partikel) von hexagonalen Kristalliten. Deren Form und Dimensionen kommen den natürlichen HAP-Kristalliten im Zahnschmelz bis auf das Fehlen einer geordneten räumlichen Anordnung sehr nahe [10]. Der Wirkstoff HAP wurde darüber hinaus unter anderem auch mittels Röntgenpulverdiffraktometrie untersucht $[13,14]$, was die Ähnlichkeit im Vergleich zu Zahnschmelz-HAP bestätigt.

Studien zu HAP im Bereich der Zahnpflege wurden in den letzten Jahren in zunehmender Anzahl von deutschen [15-23] und internationalen Autoren [24-28] verschiedener zahnmedizinischer Universitäten in begutachteten Journalen publiziert. Ergänzt werden diese Arbeiten durch interdisziplinäre Grundlagenforschung und mechanistische Studien von Universitäten und Forschungseinrichtungen wie Fraunhofer- und Max-Planck-Instituten, z.B. zu den Affinitätsparametern von HAP-Partikeln und Zahnoberflächen [10,29].

Das Fachbuch „Toothpastes“ greift HAP in 2 Kapiteln als Wirkstoff auf [30]. Es wird darauf verwiesen, dass HAP das Potenzial hat, Kariesläsionen zur remineralisieren. In diesem Zusammenhang werden unterschiedliche Primärstudien referenziert [31-34]. Darüber hinaus wird HAP als Putzkörper in Zahnpasten aufgeführt [30]. Der Vorteil von HAP als Putzkörper ist insbesondere, dass die Partikel annähernd dieselbe Härte wie der humane Zahn aufweisen und somit das Potenzial einer übermäßigen Abrasion minimiert ist (im Gegensatz zu beispielsweise Perlit oder $\mathrm{Al}_{2} \mathrm{O}_{3}$, die eine hohe relative Härte aufweisen) [35]. 
Überblick über die Verwendung von Hydroxylapatit als biomimetischem Wirkstoff in der Zahn- und Mundpflege

Synthetisch kann HAP über unterschiedliche Wege hergestellt werden [3, 36], z. B. mittels Hydrothermalsynthese aus Kalzium- und Phosphatsalzen [3, 9, 36]:

$5 \mathrm{Ca}^{2+}+3 \mathrm{PO}_{4}^{3-}+\mathrm{OH}^{-} \rightarrow \mathrm{Ca}_{5}\left(\mathrm{PO}_{4}\right)_{3}(\mathrm{OH})$

Die Charakterisierung von HAP-Wirkstoffen kann mithilfe unterschiedlicher analytischer Methoden erfolgen (z.B. Röntgenpulverdiffraktometrie und REM $[10,14])$.

In Zahnpflegeprodukten wird HAP in Deutschland bereits seit über 10 Jahren eingesetzt [20,21,30]. Erste Studien zu HAP im Bereich der Zahnpflege wurden Ende der 1980er-Jahre unter anderem von einer Arbeitsgruppe des Universitätsklinikums Gießen um Hüttemann und Dönges (Schutz vor überempfindlichen Zahnhälsen durch HAP) [23] und einer japanischen Arbeitsgruppe um Kani et al. (Kariesschutz durch HAP) [31] publiziert. Vorteilhaft bei der Verwendung von HAP ist die hohe Übereinstimmung mit den natürlichen Schmelzkristalliten sowie eine gute Verträglichkeit (d.h. eine hohe Biokompatibilität) [10,37]. So ist das Risiko unerwünschter Nebenwirkungen bei Verschlucken, wie sie z. B. bei fluoridhaltigen Produkten bekannt sind (Fluorose), nicht vorhanden. Fluoride werden häufig zur Remineralisation und zur Kariesprävention eingesetzt und es gibt zahlreiche Studien zu diesem Wirkstoff [30]. Insbesondere bei Säuglingen und Kleinkindern besteht jedoch die Gefahr einer Überdosierung bei der Verwendung einer Fluoridzahnpasta, da sie häufig einen Teil der Zahnpasta verschlucken. Besonders zum Tragen kommt dies, wenn mehrere Fluoridquellen miteinander kombiniert werden (Fluoridzahnpasta, fluoridiertes Speisesalz, Fluoridtabletten, Mineralwässer mit Fluorid etc.) [38]. Die Prävalenz von milden Fluorosen liegt in Deutschland zwischen 10 und 20\%

- Tab. 1 Übersicht über die Wirkmechanismen von HAP in der Mundhöhle (Details siehe Text) [41].

\begin{tabular}{|l|l|}
\hline Wirkmechanismen & \begin{tabular}{l} 
Beschreibungen \\
\hline physikalisch
\end{tabular} \\
$\begin{array}{l}\text { Schichtbildung durch Anlage- } \\
\text { rung von HAP-Partikeln an Zahn- } \\
\text { oberflächen, Reinigungseigen- } \\
\text { schaften als Putzkörper }\end{array}$ \\
\hline (bio-)chemisch & $\begin{array}{l}\text { Quelle für Kalzium- und Phos- } \\
\text { phationen, Bildung einer Bin- } \\
\text { dung zwischen HAP-Partikeln } \\
\text { und der Zahnoberfläche, „Opfer- } \\
\text { material“ für Säureangriffe }\end{array}$ \\
\hline biologisch & $\begin{array}{l}\text { Interaktion von HAP-Partikeln } \\
\text { mit Mikroorganismen } \rightarrow \text { Reduk- } \\
\text { tion der bakteriellen Anlagerung } \\
\text { an der Zahnoberfläche) }\end{array}$ \\
\hline
\end{tabular}

(Trinkwasser wird in Deutschland nicht fluoridiert) [38]. Ein entsprechender Hinweis auf Fluoridzahnpasten ist gemäß Europäischer Kosmetikverordnung bindend, sofern die Zahnpasta 1000-1500 ppm Fluorid enthält (außer, wenn nicht ohnehin als für Kinder ungeeignet gekennzeichnet) [39].

\section{ZUSATZINFO}

„Für Kinder bis 6 Jahre: Nur erbsengroße Menge Zahnpasta benutzen. Zur Vermeidung übermäßigen Verschluckens Zähneputzen nur unter Aufsicht. Bei zusätzlicher Aufnahme von Fluorid den Zahnarzt oder Arzt befragen.“

Ein derartiger Hinweis ist für fluoridfreie Zahnpasten mit HAP nicht notwendig. Damit stellt HAP als Wirkstoff eine Alternative zu Fluorid dar, die sich für alle Altersgruppen eignet und auch in größeren Konzentrationen eingesetzt werden kann, während die Einsatzkonzentration von Fluorid in Zahnpasten gesetzlich auf $0,15 \% \bumpeq 1500 \mathrm{ppm}$ beschränkt ist $[10,39,40]$.

Formulierungen mit HAP können in unterschiedlichen Bereichen der Mundpflege eingesetzt werden, wobei die Wirkung von HAP auf verschiedenen Wirkmechanismen basiert ( $\triangleright$ Tab. 1) $[9,41]$.

Klinische Studien zeigen unter anderem eine Schutzwirkung bei Dentinhypersensibilität [18,23, 27, 28,42,43] und die Verbesserung der Parodontalgesundheit (z. B. Reduktion von Zahnfleischbluten) $[16,17,44]$. In einer klinischen Doppelblindstudie von Harks et al. wurde an den Unikliniken Münster und Würzburg gezeigt, dass sich die Zahnfleischgesundheit von Parodontitispatienten durch die Verwendung einer HAP-Zahnpasta in gleichem Maße verbesserte wie durch die Verwendung einer fluoridhaltigen Zahnpasta mit den antibakteriellen Komponenten Amin und Zinn [17]. Die gewonnenen Plaqueproben wurden darüber hinaus von Hagenfeld et al. mit modernsten mikrobiologischen Analysemethoden (Next Generation Sequencing) untersucht [16].

Im Bereich Dentinhypersensibilität zeigt eine Metaanalyse von $\mathrm{Hu}$ et al. die Wirksamkeit von HAP-Zahnpasten im Vergleich zu Negativkontrollen [42]. Dabei wird von einem rein physikalischen, anästhetikafreien Wirkprinzip ausgegangen, wonach HAP-Partikel offenliegende Dentintubuli blockieren und damit die Einwirkung diverser Stimuli auf den Zahnnerv verhindern, was die Schmerzanfälligkeit reduziert [18, 23,43,45].

Überdies bestätigen In-situ-Studien die Reduktion der initialen bakteriellen Kolonisation an Schmelzoberflächen durch die Verwendung von HAP. Die Studien von Hannig et al. [20] und Kensche et al. [19] zeigen eine vergleich- 
bare Reduktion der bakteriellen Anlagerung an Zahnoberflächen, wie sie bei Chlorhexidin, dem Goldstandard gegen orale Biofilme [46], festgestellt werden kann. Dabei werden die Bakterien durch HAP nicht abgetötet [19], sodass das ökologische Gleichgewicht der Mundhöhle bei gleichzeitiger Senkung der Bakterienlast an der Zahnoberfläche intakt bleibt. HAP zeigt dabei keine bakteriziden Eigenschaften, sondern eine antiadhärente Wirkung mit gleicher Wirksamkeit (d. h. gleiche Reduktion der bakteriellen Anlagerung) [19]. Während klassische antibakterielle Wirkstoffe wie Chlorhexidin oder Zinnverbindungen (z. B. Zinnfluorid, Zinnchlorid) bei längerer Verwendung zu unerwünschten, häufig bräunlichen Zahnverfärbungen führen können $[47,48]$, besteht dieses Risiko bei HAP nicht. Stattdessen trägt HAP als weißer Wirkstoff tatsächlich zu einem Whitening-Effekt bei [22, 49-53].

Diese schonende Reduktion der bakteriellen Anlagerung an den Zähnen ist auch hilfreich, um Karies und Zahnfleischproblemen vorzubeugen [7,35,54]. In der Praxis lässt sich allerdings feststellen, dass selbst bei gründlicher häuslicher Zahnpflege keine vollständige Plaqueentfernung zur Karies- und Parodontitisprävention möglich ist [55]. Dies zeigen auch die aktuellen Ergebnisse der Fünften Deutschen Mundgesundheitsstudie, die belegen, dass in Deutschland - trotz Verbesserungen - Karies bei Erwachsenen allgemein noch weitverbreitet ist (durchschnittliche Karieserfahrungen: 35- bis 44-Jährige: 11,2 Zähne bei einer Kariesprävalenz von 97,5\%; 65- bis 74Jährige: 17,7 Zähne bei einer Kariesprävalenz von 99\%; 75- bis 100-Jährige: 21,6 Zähne bei einer Kariesprävalenz von 99\%) [56]. Auch bei Kindern ist in Deutschland Karies nach wie vor weitverbreitet [57].

Somit besteht neben etablierten Wirkstoffen und Wirkkonzepten nach wie vor ein hoher Bedarf an gut verträglichen (biomimetischen) Konzepten ohne Fluoroserisiko, die auch bei Anwesenheit von Plaque wirksam sind und das Remineralisations-Demineralisations-Gleichgewicht in Richtung der Remineralisation verschieben können.

\section{Wirkungen von Hydroxylapatit in der Remineralisation von Schmelz und Dentin}

\section{Klinische Studien}

Im Bereich der Kariesprävention mit HAP gibt es 2 publizierte klinische Studien. Kani et al. publizierten die 1. Studie, die den kariesprotektiven Effekt einer HAP-haltigen Zahnpasta an japanischen Schulkindern beschreibt [31]. Diese klinische Studie wird auch im Fachbuch „Toothpastes“ zitiert [30]. Da es eine Vielzahl an Studien gibt, die den kariesprotektiven Effekt von Fluoriden in Zahnpasten beschreiben $[30,58]$, wurde in einer klinischen
Studie von Schlagenhauf et al. eine fluoridfreie Zahnpasta mit HAP mit einer Zahnpasta mit 1400 ppm Fluorid (Aminfluorid und Zinn(II)fluorid) verglichen [15]. Diese Doppelblindstudie wurde an 5 deutschen Universitätskliniken (Würzburg, Regensburg, München, Dresden, Frankfurt) an 150 Probanden in kieferorthopädischer Behandlung (d.h. einer Kariesrisikogruppe) durchgeführt und im Fachmagazin Journal of Investigative and Clinical Dentistry publiziert [15]. In der gewählten Kariesrisikogruppe können erste Kariesläsionen bereits nach nur 1 Monat beobachtet werden, wie O'Reilly und Featherstone berichten [59]. Die Kariesdetektion in der Studie von Schlagenhauf et al. erfolgte nach standardisierten ICDAS-Kriterien (International Caries Detection and Assessment System [60]). Die statistische Nichtunterlegenheitsanalyse zeigte keine signifikanten Unterschiede zwischen der HAP-Gruppe und der Fluoridgruppe.

\section{In-situ-Studien}

Eine In-situ-Studie zum Schmelzremineralisationspotenzial einer HAP-Zahnpasta unter Mundhöhlenbedingungen wurde im Dezember 2019 von Amaechi und Mitarbeitern im internationalen Fachjournal BDJ Open publiziert [24]. In dieser Studie wurden eine fluoridfreie Zahnpasta mit HAP und eine Zahnpasta mit 500 ppm Fluorid (Aminfluorid) hinsichtlich der Remineralisation initialer Kariesläsionen und dem Schutz vor Demineralisation untersucht. Die Doppelblindstudie wurde mit 30 Probanden in einem Cross-over-Design durchgeführt. Die Mineraldichte und Läsionstiefe der In-situ-Schmelzprüfkörper wurden mittels Mikroradiografie quantifiziert. Beide Zahnpasten führten zu einer signifikanten Remineralisation des Schmelzes und einer Reduktion der Läsionstiefe im Vergleich zu den Ausgangswerten ( $p<0,0001)$. Eine Demineralisation von intakten Schmelzproben wurde nicht beobachtet. Dies lässt schlussfolgern, dass die untersuchte HAP-Zahnpasta in dieser Studie initiale Kariesläsionen genauso gut wie die Fluoridzahnpasta remineralisieren konnte. Die Analysen der mikroradiografischen Aufnahmen dieser In-situ-Studie zeigen zudem, dass Fluorid die Schmelzoberfläche primär oberflächlich remineralisiert, wohingegen HAP bis in tiefere Schichten des Schmelzes homogen remineralisiert [24].

Eine vorangegangene In-situ-Studie von Najibfard et al. zeigte ebenfalls eine remineralisierende Wirkung von HAP [25]. Hier wurden Zahnpasten mit 5\% und 10\% HAP sowie mit 1100 ppm Fluorid (Natriumfluorid) getestet. Alle 3 Zahnpasten erzielten eine signifikante Remineralisation, wobei es keine signifikanten Unterschiede zwischen den Gruppen gab [25].

\section{In-vitro-Studien}

Neben den oben genannten In-situ-Remineralisationsstudien wurden auch In-vitro-Remineralisationsstudien publiziert, von denen einige hier beispielhaft dargestellt werden. 
Zahnmediziner an der Charité - Universitätsmedizin Berlin zeigten in einer In-vitro-Studie, dass fluoridfreie Zahnpasten mit HAP zu höheren Remineralisationseffekten an Dentin führten als die Verwendung von Zahnpasten mit Aminfluorid [21]. In einer anderen In-vitro-Studie wurde der Remineralisationseffekt eines HAP-Gels untersucht. Dabei konnte ein signifikanter remineralisierender Effekt festgestellt werden, der deutlich ausgeprägter war als bei künstlichem Speichel alleine [61]. Dies zeigt, dass die Verwendung von HAP auch bei normalem Speichelfluss hinsichtlich der Remineralisation vorteilhaft sein kann. Auch bei reduziertem Speichelfluss (Hyposalivation) ist HAP als Wirkstoff für die Remineralisation interessant, weil er im Gegensatz zu anderen Wirkstoffen für seine Wirkweise nicht zwangsläufig auf die Kalzium- und Phosphationen aus dem Speichel angewiesen ist [62]. Eine In-vitro-Studie von de Carvalho et al. zeigt, dass eine HAP-haltige Paste einen protektiven Effekt gegen die Entwicklung einer Schmelzkaries hat [63]. Jedoch konnten Esteves-Oliveira et al. in einer In-vitro-Studie keinen vor Demineralisation schützenden Effekt durch eine HAP-Zahnpasta (im Gegensatz zu Fluoridzahnpasten) zeigen [64]. Scholz et al. demonstrierten bei der Verwendung von unterschiedlichen Fluoridgelen In vitro, dass Fluorid-Kalzium-Präzipitate nur bei Fluoridgelen mit saurem pH-Wert (und nicht bei Fluoridgelen mit neutralem $\mathrm{pH}$-Wert) beobachtet werden konnten [65]. Dies deutet darauf hin, dass hier Kalziumionen aus dem Zahnmineral gelöst und in den Remineralisatiosprozess eingebunden werden, und diese nicht aus dem Wirkstoff selbst stammen (wie bei der Verwendung von kalziumphosphathaltigen Wirkstoffen). Hornby et al. bestätigten durch die Verwendung von radioaktiv $\left({ }^{45} \mathrm{Ca}\right)$ markiertem HAP, dass Kalziumionen aus dem HAP-Wirkstoff im Remineralisationsprozess involviert sind [66].

\section{Interaktion von Hydroxylapatitpartikeln mit der Zahnoberfläche und mechanistische Untersuchungen}

Die Interaktion von HAP-Partikeln mit Zahnschmelzoberflächen wurde in einer In-vitro-Studie von Fabritius-Vilpoux et al. mittels hochaufgelöster REM untersucht [10]. HAP-Partikel lagern sich selbst in Abwesenheit jeglicher organischer Substanzen an Zahnoberflächen an und bilden Mineralbrücken zwischen Schmelz- und Partikelkristalliten [10]. Eine Anlagerung von HAP-Partikeln an Schmelzoberflächen wurde von Kensche et al. auch unter Mundhöhlenbedingungen (In situ) gezeigt [19]. Lelli et al. demonstrierten in einer Ex-in-vivo-Studie, dass sich durch die Verwendung einer HAP-Zahnpasta eine kalziumphosphathaltige Schicht auf der Zahnoberfläche bildet [67]. Die Ergebnisse einer In-vitro-Studie zum Erosionsschutz von HAP bei Säureangriffen zeigt, dass der nach Verwendung entsprechender Produkte auf dem Zahnmineral vorhandene HAP-Film als „Opferanode“ dient und die Zahn- oberfläche durch Kalzium- und Phosphationen aus Speichel und gelöstem HAP-Wirkstoff geschützt und teilweise remineralisiert wird [68]. Sudradjat et al. zeigten in einer 3-tägigen Pilotstudie unter In-vivo-Bedingungen an Kindern, dass es durch die Verwendung eines HAP-Gels in den Medianen eine Tendenz zu höheren Kalzium- und Phosphorgehalten in der Plaque gibt: Ausgangswert Kalzium: 0,25 Gew.-\%, Kalzium nach Anwendung: 0,40 Gew.-\%; $p=0,5 /$ Ausgangswert Phosphor: 1,17 Gew.-\%, Phosphor nach Anwendung: 1,41 Gew.-\%; p =0,3 [29]. Cieplik et al. untersuchten das Verhalten von HAP in bakteriellen Biofilmen in einer In-vitro-Studie [69]. Der Wirkstoff HAP setzt in sauren Streptococcus mutans-Biofilmen Kalziumionen frei. Es ist wichtig, gerade bei vorhandenen Biofilmen (z. B. an schwer zu reinigenden Flächen) zusätzlich externe Kalziumquellen bereitzustellen, weil die sogenannten Exopolysaccharide (EPS) der Bakterien Kalzium aufnehmen und damit binden [70]. Hierdurch kann das Remineralisationspotenzial des Speichels gesenkt werden. Außerdem führt die Verwendung von HAP zu einer leichten Erhöhung des Biofilm-pH-Werts [69]. Dies ist deshalb von Bedeutung, weil die Kristallisation von HAP (d. h. auch die Remineralisation aus dem Speichel) im neutralen bis leicht basischen $\mathrm{pH}$-Bereich begünstigt ist [3].

Shaw et al. zeigten überdies, dass Plaque von Kindern ohne Karieserfahrung mehr Kalzium und Phosphat enthält als Plaque von kariesanfälligen Kindern [71]. Auch deshalb erscheint die Zugabe von kalziumphosphathaltigen Wirkstoffen wie HAP in Zahnpflegeformulierungen sinnvoll zu sein.

\section{FAZIT}

Der Wirkstoff Hydroxylapatit wird in unterschiedlichen Bereichen der Zahnpflege eingesetzt [9, 15, $17-21,24,31,41,42,53]$. Studien zeigen, dass HAP die Remineralisation der Zähne begünstigt [21, 24, $25,61]$. Auf Basis der publizierten Daten lassen sich in der Remineralisation folgende Wirkmechanismen von HAP ableiten [41]:

- biophysikalisch: Anlagerung von HAP an Schmelz und Dentin

- biochemisch: lokale Auflösung, Freisetzung von Kalzium- und Phosphationen, Säurepuffer, Kristallisationskeimbildung und Wachstum

Die oben beschriebenen, publizierten In-vivo-, In-situ- und In-vitro-Studien zeigen ein zunehmend klareres Bild der Wirkweisen und Effizienz von HAP insgesamt und verdeutlichen, dass HAP auch über die Remineralisation von Schmelz und Dentin hinaus ein vielversprechender biomimetischer Wirkstoff in der Zahnpflege für Personen aller Altersstufen ist. 
WEITERFÜHRENDE LITERATUR

- Open Dent J 2019; 13: 274-287

- Bioinspir Biomim Nan 2019; 8: 141-153

- BDJ Open 2019; 5: 18

- J Invest Clin Dent 2019; 10: e12399

Danksagung

Die Autoren danken der Zahnärztin Frau Dr. med. dent. Barbara Simader für die Durchsicht des Manuskripts und der Biologin Frau Dr. rer. nat. Kathia Fabritius-Vilpoux für die REM-Analysen.

\section{Abstract}

The oral cavity is a highly dynamic environment where the de- and remineralization of tooth enamel and dentin are in equilibrium. This equilibrium can be shifted towards an undesired net-demineralization by bacterial biofilms (dental caries) or an acidic diet (dental erosion). One way to remineralize the dental hard tissues with an active ingredient is the use of particulate hydroxyapatite $\left[\mathrm{Ca}_{5}\left(\mathrm{PO}_{4}\right)_{3}(\mathrm{OH})\right]$. Hydroxyapatite (HAP) is a well-tolerated, biomimetic calcium phosphate mineral that represents a potential source of calcium and phosphate ions in an acidic environment. Studies show that hydroxyapatite particles adhere to the tooth surface. As demonstrated under oral cavity conditions (In situ), the use of HAP containing products leads to a remineralization of demineralized enamel down to deeper layers. This article summarizes scientific evidence supporting the biomimetic remineralization of tooth tissues by hydroxyapatite based on In vivo, In situ and In vitro studies.

\section{Interessenkonflikt}

J. E. und F. M. arbeiten in der wissenschaftlichen Abteilung der Firma Dr. Kurt Wolff GmbH \& Co. KG. H. O. F. und B. T.A. kooperieren mit J.E. und F. M. in einer Reihe von Forschungsprojekten zu wissenschaftlichen Grundlagen mit finanzieller Unterstützung von Dr. Wolff. Die Ergebnisse aus diesen Projekten werden regelmäßig in internationalen Fachjournalen publiziert und auf wissenschaftlichen Tagungen vorgestellt.

Autorinnen/Autoren

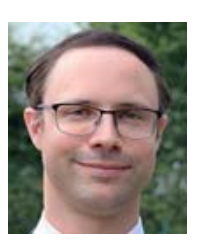

\section{Dr. Joachim Enax}

Dr. Joachim Enax promovierte 2014 in Anorganischer Chemie über die Charakterisierung von Haifischzähnen und die Entwicklung biomimetischer Kalziumphosphat-Polymer-Dentalkomposite an der Universität DuisburgEssen. Seit 2015 arbeitet er als Senior Scientist Oral Care bei der Firma Dr. Kurt Wolff GmbH \& Co. KG in Bielefeld. Er ist Autor verschiedener Publikationen im Themenfeld Zahnpflege und lehrt zusätzlich als externer Lehrbeauftragter an der Technischen Hochschule OstwestfalenLippe.

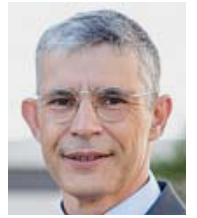

\section{Prof. Dr. Helge-Otto Fabritius}

Prof. Dr. Helge-Otto Fabritius lehrt Bionik und Materialentwicklung an der Hochschule Hamm-Lippstadt. Er promovierte 2008 in der Zentralen Einrichtung Elektronenmikroskopie der Universität Ulm im Bereich Biologie über Biomineralisation. Von 2009 bis 2018 leitete er die Arbeitsgruppe „Biologische Verbundwerkstoffe“ am Max-Planck-Institut für Eisenforschung $\mathrm{GmbH}$ in Düsseldorf. Als Experte für Zusammenhänge von Struktur, Zusammensetzung, Eigenschaften und Funktion in biogenen Materialien und deren experimenteller Charakterisierung beschäftigt er sich seit Jahren intensiv mit Zähnen und deren Struktur auf Materialebene und hat eine umfangreiche Publikationshistorie auf dem Gebiet der Biomimetik.

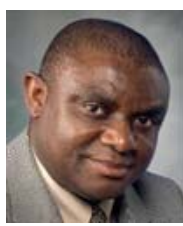

Prof. Dr. Bennett T. Amaechi

Prof. Dr. Bennett T. Amaechi, BDS, MS, PhD, MFDS RCPS (Glasg), FADI, ist Professor und Direktor für Kariologie am Institut für Zahnmedizin an der University of Texas Health San Antonio/USA. Nach seinem Abschluss an der Obafemi Awolowo University, Nigeria, setzte Dr. Amaechi seine Laufbahn als Zahnmediziner in Europa und den USA fort. Er erhielt einen Master in Prothetik am Guy's Hospital, London/UK, und im Anschluss den Doktortitel in Kariologie und präventiver Zahnmedizin an der University of Liverpool, UK. Seit 2001 ist er Direktor für Kariologie an der University of Texas Health San Antonio/USA. Er ist Autor zahlreicher wissenschaftlicher Fachpublikationen, einem Buch über dentale Erosionen sowie Buchkapiteln und Konferenzbeiträgen.

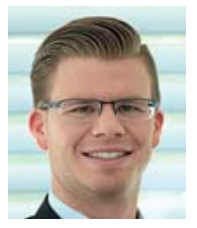

\section{Dr. Frederic Meyer}

Dr. Frederic Meyer promovierte 2017 in Mikrobiologie und Epidemiologie über die Epidemiologie und Mikrobiomzusammensetzung von frühkindlicher Karies an der TU Braunschweig. Bereits während seines Studiums spezialisierte sich Dr. Meyer auf die orale Biologie. Seit 2017 arbeitet er als Senior Scientist Oral Care bei der Firma Dr. Kurt Wolff GmbH \& Co. KG in Bielefeld. Er ist Autor unterschiedlicher Publikationen aus den Bereichen der oralen Biologie und Zahnpflege.

\section{Korrespondenzadresse}

\author{
Dr. Joachim Enax \\ Dr. Kurt Wolff GmbH \& Co. KG \\ Research Department \\ Johanneswerkstraße 34-36 \\ 33611 Bielefeld \\ Tel.: + 49(0)52188086064 \\ joachim.enax@drwolffgroup.com
}

Literatur

[1] Teaford MF, Smith MM, Ferguson MWJ. Development, function and evolution of teeth. Cambridge: Cambridge University Press; 2000

[2] Lowenstam HA, Weiner S. On Biomineralization. New York, London: Oxford University Press; 1989 
[3] Dorozhkin SV, Epple M. Biological and medical significance of calcium phosphates. Angew Chem Int Ed 2002; 41: 31303146

[4] Beniash E, Stifler CA, Sun CY et al. The hidden structure of human enamel. Nat Commun 2019; 10: 4383

[5] Fejerskov O, Kidd E. Dental caries: The disease and its clinical management. Oxford: Wiley; 2009

[6] Dawes C. What is the critical $\mathrm{pH}$ and why does a tooth dissolve in acid? J Can Dent Assoc 2003; 69: 722-724

[7] Epple M, Enax J. Moderne Zahnpflege aus chemischer Sicht. Chem Unserer Zeit 2018; 52: 218-228

[8] Meyer F, Amaechi BT, Fabritius $\mathrm{HO}$ et al. Overview of calcium phosphates used in biomimetic Oral care. Open Dent J 2018; 12: 406-423

[9] Enax J, Epple M. Synthetic hydroxyapatite as a biomimetic oral care agent. Oral Health Prev Dent 2018; 16: 7-19

[10] Fabritius-Vilpoux K, Enax J, Herbig M et al. Quantitative affinity parameters of synthetic hydroxyapatite and enamel surfaces in vitro. Bioinspir Biomim Nan 2019; 8: 141-153

[11] Fabritius HO, Meyer F, Enax J. Biomimetik - Die Natur als Vorbild. Spektrum Wiss 2018; 12: 46-53

[12] Hannig M, Hannig C. Nanomaterials in preventive dentistry. Nat Nanotechnol 2010; 5: 565-569

[13] Enax J, Fabritius HO, Fabritius-Vilpoux K et al. Physicochemical properties of hydroxyapatite-microclusters used in oral care (poster). IADR/PER General Session, London, England, 2018. Im Internet: https://iadr2018.zerista.com/event/member/ 492940; Stand: 13.05 .2020

[14] Peetsch A, Epple M. Characterization of the solid components of three desensitizing toothpastes and a mouth wash. Materialwiss Werkstofftech 2011; 42: 131-135

[15] Schlagenhauf U, Kunzelmann KH, Hannig C et al. Impact of a non-fluoridated microcrystalline hydroxyapatite dentifrice on enamel caries progression in highly caries-susceptible orthodontic patients: A randomized, controlled 6-month trial. J Invest Clin Dent 2019; 10: e12399

[16] Hagenfeld D, Prior K, Harks I et al. No differences in microbiome changes between anti-adhesive and antibacterial ingredients in toothpastes during periodontal therapy. J Periodont Res 2019; 54: 435-443

[17] Harks I, Jockel-Schneider Y, Schlagenhauf $U$ et al. Impact of the daily use of a microcrystal hydroxyapatite dentifrice on de novo plaque formation and clinical/microbiological parameters of periodontal health. A randomized trial. PLoS One 2016; 11: e0160142

[18] Hiller KA, Buchalla W, Grillmeier I et al. In vitro effects of hydroxyapatite containing toothpastes on dentin permeability after multiple applications and ageing. Sci Rep 2018; 8: 4888

[19] Kensche A, Holder C, Basche S et al. Efficacy of a mouthrinse based on hydroxyapatite to reduce initial bacterial colonisation in situ. Arch Oral Biol 2017; 80: 18-26

[20] Hannig C, Basche S, Burghardt T et al. Influence of a mouthwash containing hydroxyapatite microclusters on bacterial adherence in situ. Clin Oral Investig 2013; 17: 805-814

[21] Tschoppe P, Zandim DL, Martus P et al. Enamel and dentine remineralization by nano-hydroxyapatite toothpastes. J Dent 2011; 39: 430-437

[22] Jin J, Xu X, Lai G et al. Efficacy of tooth whitening with different calcium phosphate-based formulations. Eur ] Oral Sci 2013; 121: $382-388$
[23] Hüttemann RW, Dönges H. Untersuchungen zur Therapie überempfindlicher Zahnhälse mit Hydroxylapatit. Dtsch Zahnärztl Z 1987; 42: 486-488

[24] Amaechi BT, AbdulAzees PA, Alshareif DO et al. Comparative efficacy of a hydroxyapatite and a fluoride toothpaste for prevention and remineralization of dental caries in children. BDJ Open 2019; 5: 18

[25] Najibfard K, Ramalingam K, Chedjieu I et al. Remineralization of early caries by a nano-hydroxyapatite dentifrice. J Clin Dent 2011; 22: 139-143

[26] Vano M, Derchi G, Barone A et al. Reducing dentine hypersensitivity with nano-hydroxyapatite toothpaste: a double-blind randomized controlled trial. Clin Oral Investig 2018; 22: 313320

[27] Orsini G, Procaccini M, Manzoli L et al. A 3-day randomized clinical trial to investigate the desensitizing properties of three dentifrices. J Periodontol 2013; 84: 65-73

[28] Orsini G, Procaccini M, Manzoli L et al. A double-blind randomized-controlled trial comparing the desensitizing efficacy of a new dentifrice containing carbonate/hydroxyapatite nanocrystals and a sodium fluoride/potassium nitrate dentifrice. J Clin Periodontol 2010; 37: 510-517

[29] Sudradjat H, Meyer F, Loza K et al. In vivo effects of a hydroxyapatite-based oral care gel on the calcium and phosphorus levels of dental plaque. Eur J Dent 2020. doi:10.1055/s-00401708456

[30] van Loveren C. Toothpastes. Basel: Karger; 2013

[31] Kani K, Kani M, Isozaki A et al. Effect of apatite-containing dentifrices on dental caries in school children. J Dent Health 1989; 19: 104-109

[32] Lv K, Zhang J, Meng $X$ et al. Remineralization effect of the nano-HA toothpaste on artificial caries. Key Eng Mater 2007; 330-332: 267-270

[33] Kim MY, Kwon HK, Choi CH et al. Combined effects of nanohydroxyapatite and $\mathrm{NaF}$ on remineralization of early caries lesion. Key Eng Mater 2007: 330-332: 1347-1350

[34] Huang SB, Gao SS, Yu HY. Effect of nano-hydroxyapatite concentration on remineralization of initial enamel lesion in vitro. Biomed Mater 2009; 4: 034104. doi:10.1088/1748-6041/4/3/ 034104

[35] Enax J, Epple M. Die Charakterisierung von Putzkörpern in Zahnpasten. Dtsch Zahnärztl Z 2018; 73: 100-108

[36] Brown PW, Constantz B. Hydroxyapatite and related materials. Boca Raton: CRC Press; 1994

[37] Epple M. Review of potential health risks associated with nanoscopic calcium phosphate. Acta Biomater 2018; 77: 1-14

[38] Bundesinstitut für Risikobewertung. Für gesunde Zähne: Fluorid-Vorbeugung bei Säuglingen und Kleinkindern. 2018. doi:10.17590/20180531-085715-0

[39] Verordnung (EG) Nr. 1223/2009 des Europäischen Parlaments und des Rates vom 30. November 2009 über kosmetische Mittel; 2009. Im Internet: https://eur-lex.europa.eu/LexUriServ/ LexUriServ.do?uri=OJ:L:2009:342:0059:0209:DE:PDF; Stand: 29.04.2020

[40] Meyer F, Enax J. Early childhood caries: Epidemiology, aetiology, and prevention. Int J Dent 2018; 2018: 1-7

[41] Enax J, Fabritius HO, Fabritius-Vilpoux K et al. Modes of action and clinical efficacy of particulate hydroxyapatite in preventive oral health care - state of the art. Open Dent J 2019; 13 : 274-287

[42] Hu ML, Zheng G, Zhang YD et al. Effect of desensitizing toothpastes on dentine hypersensitivity: A systematic review and meta-analysis. J Dent 2018; 75: 12-21 
[43] Gillam DG. Dentine hypersensitivity: Advances in diagnosis, management, and treatment. New York: Springer International Publishing; 2015

[44] Hegazy SA, Salama IR. Antiplaque and remineralizing effects of Biorepair mouthwash: A comparative clinical trial. Pediatr Dent J 2016; 26: 89-94

[45] Meyer F, Fabritius HO, Enax J. Spezielle Zahnpflege bei Dentinhypersensibilität. ZMK 2017; 33: 865-868

[46] Jones CG. Chlorhexidine: is it still the gold standard? Periodontol 2000 1997; 15: 55-62

[47] Frese C, Wohlrab T, Sheng L et al. Clinical effect of stannous fluoride and amine fluoride containing oral hygiene products: A 4-year randomized controlled pilot study. Sci Rep 2019; 9: 7681

[48] Brading MG, Marsh PD. The oral environment: the challenge for antimicrobials in oral care products. Int Dent J 2003; 53 : 353-362

[49] Epple M, Meyer F, Enax J. A critical review of modern concepts for teeth whitening. Dent J (Basel) 2019; 7: 79

[50] Niwa M, Sato T, Li W et al. Polishing and whitening properties of toothpaste containing hydroxyapatite. J Mater Sci Mater Med 2001; 12: 277-281

[51] Aoki H, Matsuda K, Aoki H, Daisaku T, Sato T, Niwa M. Clinical Study of Teeth whitening Properties of Toothpastes containing Hydroxyapatite. In: LeGeros RZ, LeGeros JP, eds. Bioceramics Volume 11. Proceedings of the 11th International Symposium on Ceramics in Medicine: New York City, New York, USA, 5-8 November 1998. Singapore, New Jersey, London, Hong Kong: World Scientific; 1998: 575-577

[52] Bommer C, Flessa HP, Xu X et al. Hydroxyapatite and self-assembling peptide matrix for non-oxidizing tooth whitening. J Clin Dent 2018; 29: 57-63

[53] Dabanoglu A, Wood C, Garcia-Godoy F et al. Whitening effect and morphological evaluation of hydroxyapatite materials. Am J Dent 2009; 22: 23-29

[54] Meyer F, Enax J. Die Mundhöhle als Ökosystem. Biol Unserer Zeit 2018; 1: 62-68

[55] Deinzer R, Schmidt R, Harnacke D et al. Finding an upper limit of what might be achievable by patients: oral cleanliness in dental professionals after self-performed manual oral hygiene. Clin Oral Investig 2018; 22: 839-846

[56] Jordan AR, Micheelis W. Fünfte Deutsche Mundgesundheitsstudie (DMS V). Köln: Institut der Deutschen Zahnärzte; 2016. Im Internet: https://www.idz.institute/publikationen/ buecher/fuenfte-deutsche-mundgesundheitsstudie-dms-v. html; Stand: 29.04.2020

[57] Deutsche Arbeitsgemeinschaft für Jugendzahnpflege. Epidemiologische Begleituntersuchungen zur Gruppenprophylaxe 2016. Im Internet: https://www.daj.de/Studien.29.0. html; Stand: 29.04.2020

[58] Walsh T, Worthington HV, Glenny AM et al. Fluoride toothpastes of different concentrations for preventing dental caries. Cochrane Database Syst Rev 2019; (3): CD007868
[59] O’Reilly MM, Featherstone JD. Demineralization and remineralization around orthodontic appliances: an in vivo study. Am J Orthod Dentofacial Orthop 1987; 92: 33-40

[60] Ismail Al, Sohn W, Tellez M et al. The International Caries Detection and Assessment System (ICDAS): an integrated system for measuring dental caries. Community Dent Oral Epidemiol 2007; 35: 170-178

[61] Amaechi BT, AbdulAzees PA, Enax J et al. Hydroxyapatite vs. fluoride gel for remineralization of caries lesion: an in vitro comparison. ORCA congress, Cagliari, Italy 2020. Abstract accepted.

[62] Meyer F, Enax J. Demografische Entwicklung und häusliche Zahnpflege. ZWR - Das Deutsche Zahnärzteblatt 2018; 127: 98-104

[63] de Carvalho FG, Vieira BR, Santos RL et al. In vitro effects of nano-hydroxyapatite paste on initial enamel carious lesions. Pediatr Dent 2014; 36: 85-89

[64] Esteves-Oliveira M, Meyer-Lueckel H, Wierichs RJ et al. Cariespreventive effect of anti-erosive and nano-hydroxyapatitecontaining toothpastes in vitro. Clin Oral Investig 2017; 21 : 291-300

[65] Scholz KJ, Federlin M, Hiller KA et al. EDX-analysis of fluoride precipitation on human enamel. Sci Rep 2019; 9: 13442

[66] Hornby K, Evans M, Long $M$ et al. Enamel benefits of a new hydroxyapatite containing fluoride toothpaste. Int Dent J 2009; 59: 325-331

[67] Lelli M, Marchetti M, Foltran I et al. Remineralization and repair of enamel surface by biomimetic Zn-carbonate hydroxyapatite containing toothpaste: a comparative in vivo study. Front Physiol 2014; 5: 333

[68] Fabritius-Vilpoux K, Enax ], Meyer F et al. In vitro microstructural analysis of erosion protection by a hydroxyapatite-gel (poster). IADR/AADR/CADR General Session, Washington, USA, 2020. Im Internet: https://iadr.abstractarchives.com/ abstract/20iags-3287360/in-vitro-microstructural-analysis-oferosion-protection-by-a-hydroxyapatite-gel; Stand: 29.04.2020

[69] Cieplik F, Rupp CM, Hirsch S et al. $\mathrm{Ca}^{2+}$ release and buffering effects of synthetic hydroxyapatite following bacterial acid challenge. BMC Oral Health 2020; 20: 85

[70] Astasov-Frauenhoffer M, Varenganayil MM, Decho AW et al. Exopolysaccharides regulate calcium flow in cariogenic biofilms. PLoS One 2017; 12: e0186256

[71] Shaw L, Murray J], Burchell CK et al. Calcium and phosphorus content of plaque and saliva in relation to dental caries. Caries Res 1983; 17: 543-548

Bibliografie

DOI https://doi.org/10.1055/a-1167-4888

ZWR - Das Deutsche Zahnärzteblatt 2020; 129: 277-283

(c) Georg Thieme Verlag KG Stuttgart · New York

ISSN 0044-166X

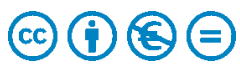

